\title{
High-temperature configurations of dimers in Si (001) surface layers
}

\author{
A.E. Kiv ${ }^{1}$, T.I. Maksymova ${ }^{2}$, N.V. Moiseenko ${ }^{2}$, V.N. Soloviev ${ }^{2}$ \\ ${ }^{1}$ Ben-Gurion University of the Negev, P. O. Box 653, Beer-Sheva, 84105, Israel \\ ${ }^{2}$ Pedagogical University, 54 Gagarin's Prospect, Krivoy Rog, 55008 Ukraine
}

\begin{abstract}
Molecular Dynamics (MD) simulation of Si (001) surface layers was performed. In the modified algorithm of MD the potential is corrected on each step of calculation. The corrections account the re-hybridization of chemical bonds in relaxation processes. It was found that the high-temperature relaxation of Si surface layers leads to formation of QuasiDisordered Phase (QDP). QDP is spread from the first layer up to the forth-fifth layer. In all these layers dimers are formed. Their characteristics (length distribution and space orientation) change from layer to layer. They differ significantly from dimers formed at lower temperatures.
\end{abstract}

Keywords: silicon surface, molecular dynamics simulation, quasi-disordered phase.

Paper received 12.03.03; accepted for publication 18.03.03.

\section{Introduction}

The experimental data show that at room temperature the dimers in the top Si (001) surface layers appear symmetric in scanning tunneling microscopy topographic images [1]. At low temperatures $(T<120 \mathrm{~K})$ dimers appear tilted [2]. A transition is expected from the low temperature ordered phase to a disordered phase above a transition temperature estimated between 200 and $120 \mathrm{~K}$. The energy difference with respect to the symmetric (saddle point) configuration is only $0.09 \mathrm{eV}$ per dimer [3]. The small energy barrier is explained by the time averaging over thermally activated flips between dimer configurations tilted in opposite directions. Some authors suggest that the semi-empirical calculation methods can predict the flip rate incorrectly and give results that differ radically from those obtained by ab initio approach (see, for example [4]). However, a model potential study of the $\mathrm{Si}(001) 2 \times 1$ surface shows that the MD simulation of various dimerization models with Stillinger-Weber (SW) potential and the total electronic energy calculations results in close characteristics of dimers [5].

Recently we studied the relaxation processes in $\mathrm{Si}$ (001) surface layers [6]. We found that at elevated temperatures $(T>600 \mathrm{~K})$ the QDP is formed. QDP and a-Si have similar structural characteristics. One of the important features inherent to these structures is existence of dangling and stressed bonds [6]. Appearance of QDP is caused by properties of the disordered diamond-like lattice $[7,8]$. In [9] is shown that in disordered Si lattice (Si clusters with distorted tetrahedral angles) three types of covalent bonds arise. The weakest bonds can be easily broken due a pre-dissociation. Thus, dangling bonds appear. A truncated $\mathrm{Si}(001)$ surface contains many dangling bonds and the system tends to minimize its energy by reconstruction of its surface. This reconstruction causes dimers to appear in the surface layers. Surface atoms move towards each other to form pairs. The dimers can arrange themselves in various patterns on the surface, and thus many reconstructions of the surface are possible. Formation of disordered dimer structures is one of important features of reconstruction of Si surface at elevated temperatures. A scanning tunneling microscope study shows the dynamics of step edges on the $\mathrm{Si}(001)$ $(2 \times 1)$ reconstructed surface at temperatures from 520 to $700 \mathrm{~K}$ [10]. It was found that mass transport at these temperatures is dominated by kink diffusion. In [11] the diffusion of $\mathrm{Si}$ dimer on $\mathrm{Si}$ (001) surface was investigated. It was concluded that a dimer could convert to a configuration that is centered between two dimer rows. 


\section{A.E. Kiv et al.: High-temperature configurations of ...}

Further we present the results of MD simulation of the relaxed processes in $\mathrm{Si}$ (001) surface layers at elevated temperatures, which lead to formation of dimers, and describe the main characteristics of dimers in connection with a structure of QDP. In section 2 the calculation details and simulation results will be described. Section 3 is devoted to discussion of results.

\section{Simulation method and results}

MD method was applied in its standard form [12], i.e., the equations of motion were solved by using the central difference scheme. The time-step was $10^{-14} \mathrm{~s}$. The usual MD algorithm was changed to account the specific features of the dynamics of covalent structures. At each step of calculations we estimated the changes of hybridization coefficients (HC) and obtained new wave functions. This allows to correct the potential and to find new coordinates at each step of simulation using the potential improved at the previous step. A new MD algorithm is schematically shown below:

The scheme for the new MD algorithm

$$
\begin{array}{lll}
X_{i}(0), V_{i}(0), F_{i}(0) & \rightarrow & X_{i}(\tau), V_{i}(\tau), \\
X_{i}(\tau), F_{i}(\tau) & \rightarrow & \text { new } \mathrm{CH} \text { and } \psi(\tau), \\
\psi(\tau) & \rightarrow & \Delta U_{1}, \\
U(0)+\Delta U_{1} & \rightarrow & F_{i}(\tau), \\
X_{i}(\tau), V_{i}(\tau), F_{i}(\tau) & \rightarrow & X_{i}(2 \tau), V_{i}(2 \tau), \text { etc. }
\end{array}
$$

Here $X_{i}(\tau), V_{i}(\tau), F_{i}(\tau)$ are coordinates, velocities and forces of $\mathrm{i}$-th atom at the moment $\tau, \psi(\tau)$ is the $\mathrm{sp}^{3}$-hybrid wave function at the moment $\tau$ and $U(0), \Delta U_{1}$ are the initial potential and the first correction to the potential.

Another modification of the MD algorithm is undertaken to describe disordered covalent structures and to account the re-bonding of atoms [8]. When the relaxation is accompanied by large distortion of covalent structures, stressed bonds appear, which are subjected with a large probability to pre-dissociation. New bonds are formed in accordance with new atomic configurations. Two conditions are satisfied in the re-bonding process:

- Each atom in the distorted covalent structure is connected with its four nearest neighbors.

- The angles aspire to have values inherent to the tetrahedral structure $\left(\sim 109^{\circ}\right)$.

Above conditions result in the case when during the re-bonding process not all bonds are restored and dangling bonds created.

A parallelepiped containing 864 atoms was chosen as a starting configuration for simulation (12 layers with 72 atoms in each one). Periodic boundary conditions were applied in two dimensions. In simulations SW potential was used with quantum corrections as mentioned above. The temperature of the system was introduced according to the MD scheme [12].

As a result of simulations, we observed formation of dimers at various locations in the $\mathrm{Si}(001)$ surface layers. The images of dimer chains at $1000 \mathrm{~K}$ are shown in Fig. 1. We did not constrain the atoms to conform to any ener- getically favorable configurations. One can see in Fig. 1 that the breaking of the dimer chains takes place, which is linked to thermal activation of surface atom migration at elevated temperatures [3]. In our model, we observed as a rule 28-36 dimers in the first layer. Most of them is oriented in the [011] direction. Some of dimers are oriented in the direction [001]. In the second layer 7-8 dimers appear. The half of them has [011] direction and the other half is oriented in direction [0O1]. In the third layer only three-four dimers are formed. Their orientation is [0O1]. In the fourth layer, we observed 2-3 dimers with the same orientation.

The average dimer length is $2.345 \AA$. In Fig. 2 we display the length distribution of dimers in each of four top layers. In Table 1 the limit lengths of dimers are presented for each layer.

Table 1. The limit lengths of dimers $\left(d_{\max }, d_{\min }, \AA\right)$

\begin{tabular}{lll}
\hline \hline Layer & $d_{\max }$ & $d_{\min }$ \\
\hline 1 & 2.17 & 2.49 \\
\hline 2 & 2.19 & 2.39 \\
\hline 3 & 2.19 & 2.44 \\
\hline 4 & 2.21 & 2.51 \\
\hline \hline
\end{tabular}

The second layer is characterized by the smallest straggling of dimer lengths. The shortest dimers are observed in the third layer.

We observe the straggling of angles of the dimer buckling $\alpha$ between the direction of dimer and the primary orientation of dimers [011]. Fig. 3 shows that dimers can appear both in [011] and opposite [ $0 \overline{1} 1]$ directions. It is found that in the first layer for the most of dimers the angle $\alpha$ is small. For the fourth layer the angle $\alpha$ is close to $90^{\circ}$. For the second and the third layers the angle $\alpha$ is distributed in the $\left(0-90^{\circ}\right)$ interval more or less uniformly.

In Fig. 4 we demonstrate a spatial orientation of dimers. On the vertical axis shown is the percentage of the total number of dimers corresponding to the given tilt angle to the surface plane (001). $\beta$ is the tilt angle.

One can see that the essential part of dimers in the first surface layer do not dispose in the plane (001). This part of dimers is characterized by stressed bonds that are unstable and can be broken as a consequence of small perturbations. We studied the dimer structure in the temperature interval 200 to $1200 \mathrm{~K}$. It was found that the distribution of angles depends on the temperature, as we demonstrate in Table 2. In this table shown is the upper limit of the angles $\alpha$ and $\beta$ of the surface dimers at different temperatures.

Table 2. The upper limit of the angle of dimer buckling $\alpha$ and of tilt $\boldsymbol{\beta}$ of dimers in the first layer.

\begin{tabular}{lllll}
\hline \hline$T, \mathrm{~K}$ & 300 & 600 & 1000 & 1200 \\
\hline$\alpha<$ & $8^{\circ}$ & $10^{\circ}$ & $15^{\circ}$ & $20^{\circ}$ \\
\hline$\beta<$ & $10^{\circ}$ & $15^{\circ}$ & $20^{\circ}$ & $25^{\circ}$ \\
\hline \hline
\end{tabular}




\section{A.E. Kiv et al.: High-temperature configurations of ...}
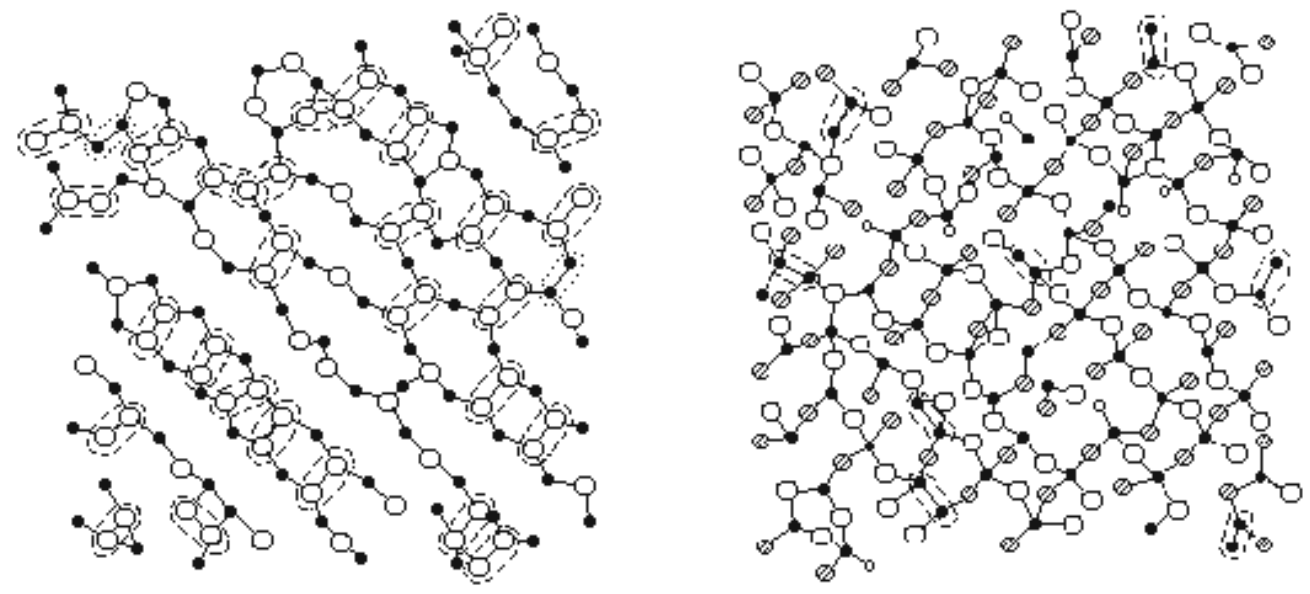

$\searrow_{[011]}^{[0 \overline{1} 1]}$
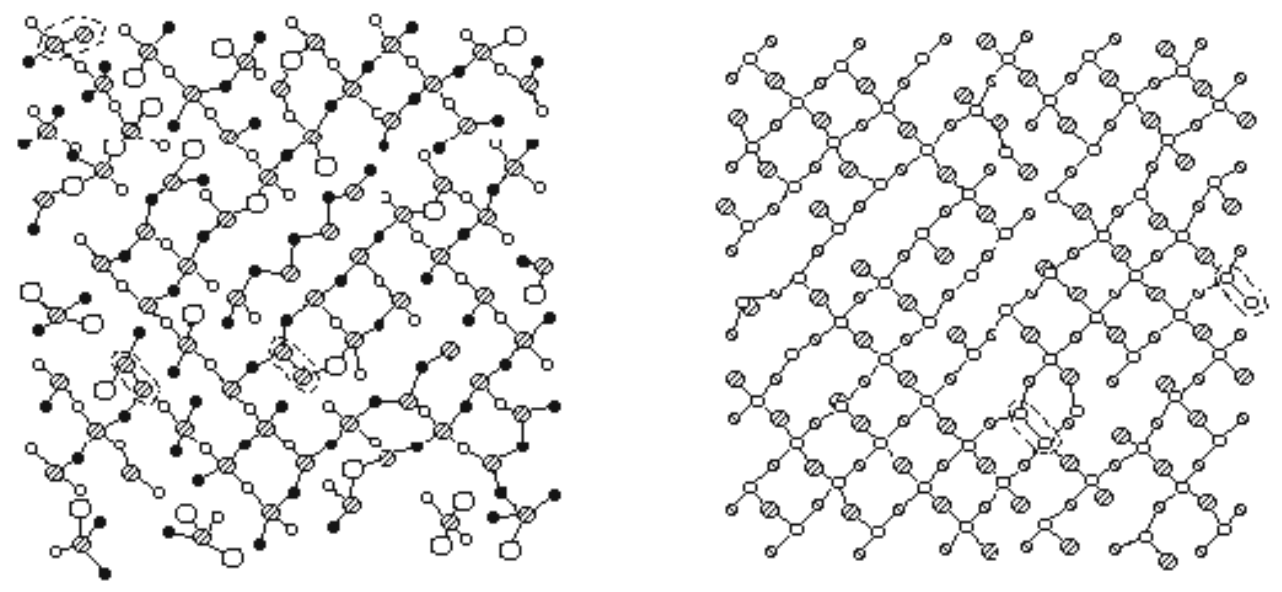

$c$

$d$

Fig. 1. Dimers in QDP at elevated temperature relaxation. The dimers are shown by dotted lines. $a$ - dimers in the first layer; $b$-dimers in the second layer; $c$-dimers in the third layer; $d$-dimers in the fourth layer.

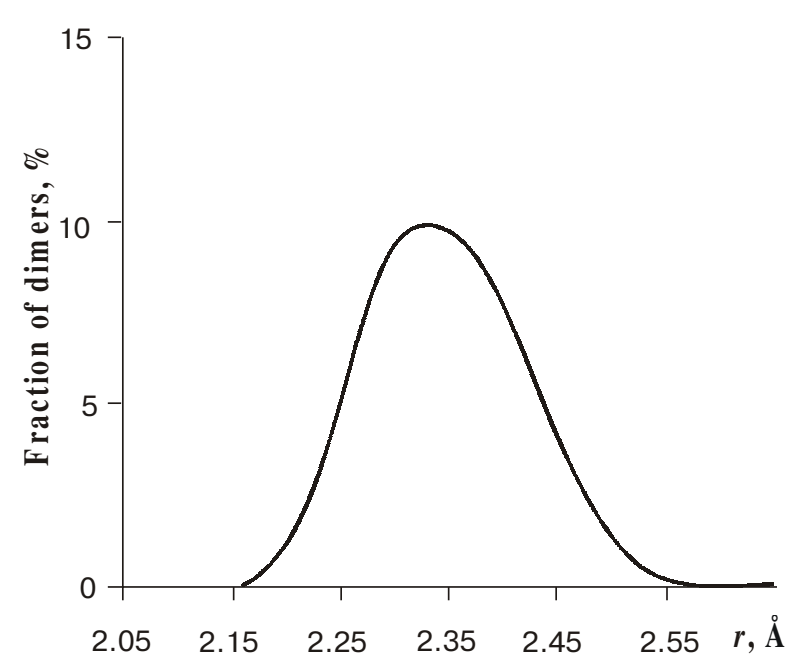

Fig. 2. The length distribution of dimers in QDP at the temperature $1000 \mathrm{~K}$.

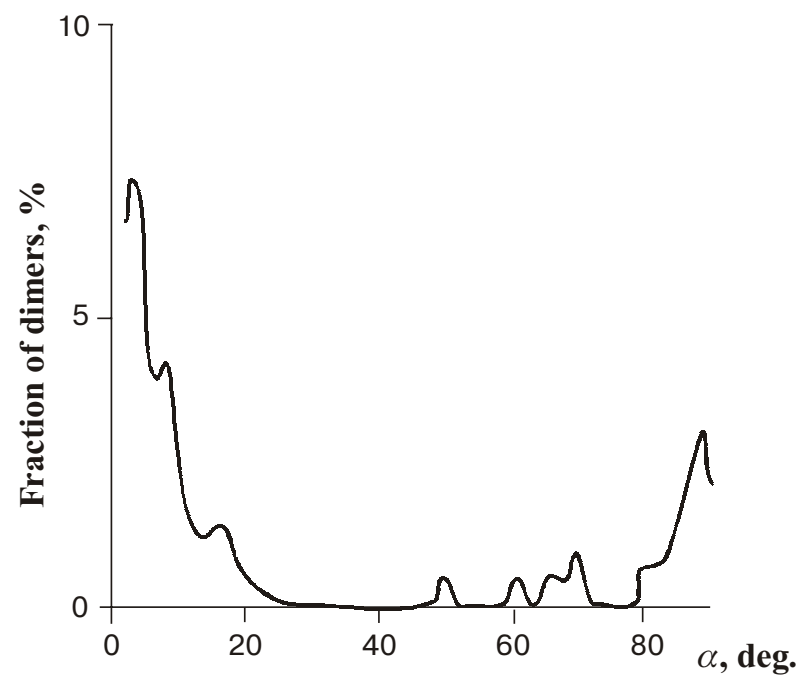

Fig. 3. The distribution of dimer buckling angles $\alpha$ in QDP at the temperature $1000 \mathrm{~K}$. 


\section{A.E. Kiv et al.: High-temperature configurations of ...}
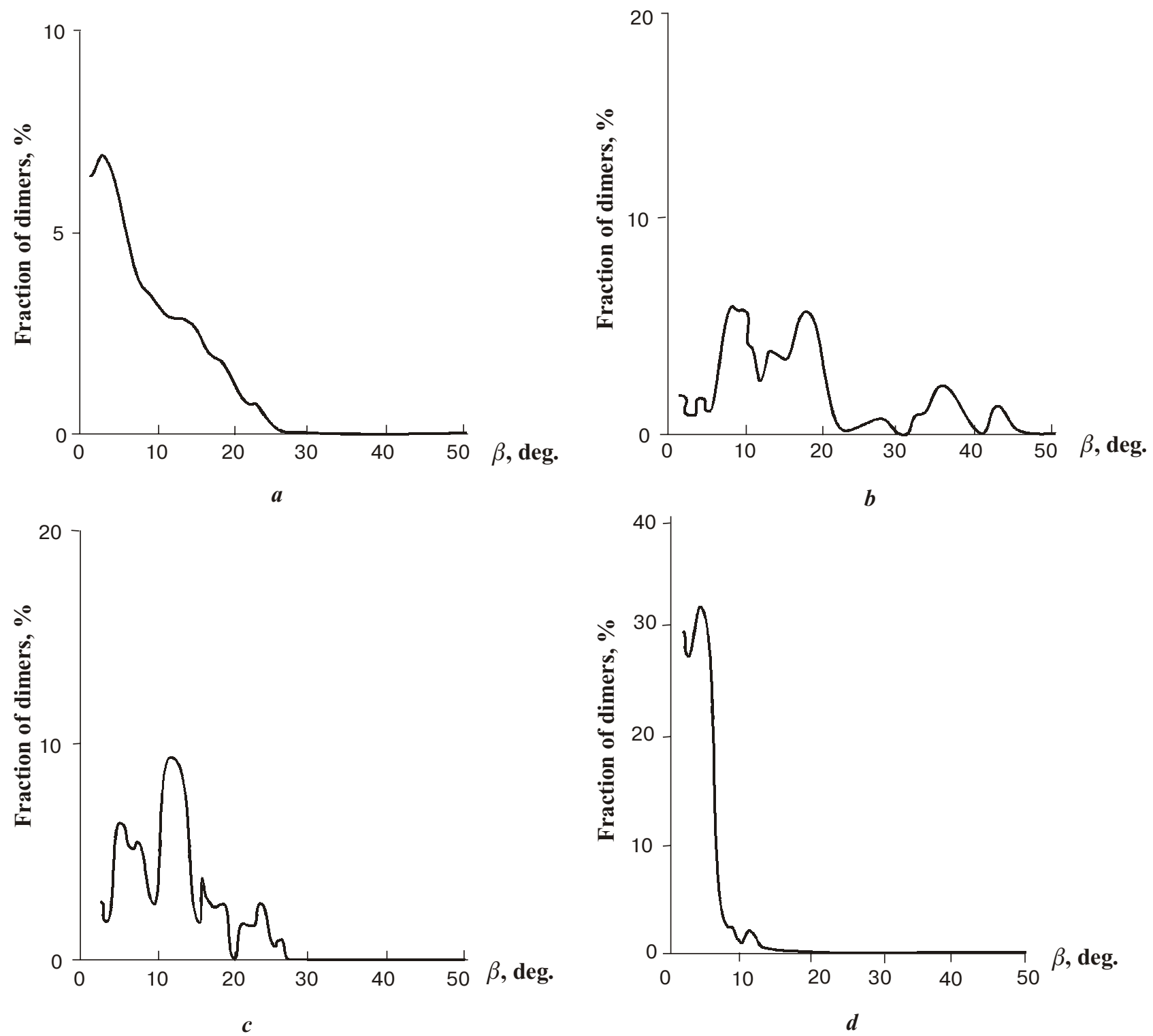

Fig. 4. The distribution of the dimer tilt angles $\beta$ at the temperature 1000 K. $a$ - the first layer; $b$-the second layer; $c$ - the third layer; $d$ - the fourth layer.

\section{Discussion}

In [6-8] we described the features of diamond-like structures and a formation of QDP in Si surface layers at elevated temperatures. Appearance of dangling bonds in the process of relaxation of the truncated $\mathrm{Si}$ crystal causes formation of dimer configurations in surface layers. It is associated with a deformation of ideal tetrahedrons and formation of stressed bonds. These bonds can be easily broken due to small energy fluctuations. In such a situation, different metastable dimer configurations are possible. A transition from one to an other dimer configuration with rather small activation energy is observed.

Experimental data show the non-monotonous temperature dependence of dimer configurations in Si surface layers. At relatively small temperatures $(T<120 \mathrm{~K})$ dimers appear tilted [2]. A transition is predicted from the low temperature dimer configurations to symmetric configurations, corresponding approximately to $200 \mathrm{~K}$. The activation energy for transition to the symmetric configuration is $0.09 \mathrm{eV}$ per dimer. The small energy barrier is explained by the time averaging over thermally activated flips between dimer configurations tilted in opposite directions [3].

Our simulation results show that the further increase of the temperature (900-1200 K) leads to essential disordering of Si surface layers and to formation of metastable QDP with different types of dimer configurations. The number of dimers in each layer correlates with the number of dangling bonds that appear in the process of relaxation of Si surface [6]. Formation of dimers ensures a relative stability of surface layers and a transition from the surface disturbed chemical bonding to the normal chemical bonds in the crystal bulk. In our simulation, we found 


\section{A.E. Kiv et al.: High-temperature configurations of ...}

that in the fifth-sixth layer the length of chemical bonds corresponds to the bulk value $2.35 \AA$

The images of dimers presented in Fig. 1 are dimer configurations that correspond to different points in the diffusion transition from one to another equilibrium position. This is a reason of a high straggling of dimer characteristics $(\alpha, \beta$ and $d)$. In [13] a piecewise dimer diffusion and the dynamics of $\mathrm{Si}(001)$ step were studied. The intensive surface mass transport on Si $(001)-(2 \times 1)$ surface at the temperatures from 520 to $700 \mathrm{~K}$ is found. Thus the high-temperature dimer configurations on $\mathrm{Si}(001)$ surface are a high mobile system, which depends strongly on the temperature changes and can influence significantly the wide region of surface processes.

\section{Conclusion}

A detailed study of characteristics of the high-temperature dimers in $\mathrm{Si}$ (001) surface layers is performed. It is found that space configurations of dimers change from the one layer to another and ensure the relative stability of surface layers. The features of the high-temperature dimers are associated with properties of QDP formed in the process of Si surface relaxation. The characteristics of dimers as well as parameters of QDP are determined strongly by temperature of the system.

\section{References}

1. Hamers R.J., Tromp R. M., and Demuth J.E. Phys. Rev. B34 5343 (1986).

2. Tabeta T., Aruga T. and Murata U. Surf. Sci. 179, L63 (1987).

3. Dabrowski J. and Scheffler M. Appl. Surf. Sci. 15, p. 58-56 (1992)

4. Gruko J. and Allen R. E. Ultramicroscopy 793, p. 42-44 (1992)

5. Abraham F.F. and Batra I.P. Surf. Sci., 163, L752 (1985).

6. Kiv A.E., Maximova T.I. and Soloviev V.N. MD simulation of the ion-stimulated processes in Si surface layers, in M.-I Baraton and I.V. Uvarova (eds), Functional Gradient Materials and Surface Layers Preparated by Fine Particles (2001).

7. Kiv A.E., Soloviev V.N., Maximova T.I. Microstructure of the Relaxed (001) Si surface, Semiconductor Physics, Quantum electronics\&Optoelectronics 3, 195-200 (2000).

8. Jacobs P.W., Kiv A.E., Balabay R.M., Grischenko N.V. Atomic configurations in $\alpha$-Si obtained by ion implantation, $C M \& N T$ 2, p. 15-20 (1998).

9. Eguchi H., Tsumuraya K., Nagano T., Kihara S. Materials Transitions, JIM, 40, 1198 (1999).

10. Pearson C., Borovsky B., Krueger M., Curtis R. and Ganz E. Phys. Rev. Lett. 74, 2710 (1995).

11. Borovsky B. Krueger M., Ganz E. Phys. Rev. Lett 22, 4229 (1997).

12. Frenkel Daan and Smit Berend Understanding Molecular Simulation, Academic Press, New-York (1966).

13. Borovsky B., Krueger M., Ganz E. Phys. Rev. B59, 1598 (1999). 